OPEN ACCESS

Edited by: Alma Balestrazzi,

University of Pavia, Italy

Reviewed by:

Michaela Griesser,

University of Natural Resources and

Life Sciences, Austria

Claudio Pastenes,

Universidad de Chile, Chile

${ }^{*}$ Correspondence:

Giovanni B. Tornielli

giovannibattista.tornielli@univr.it

Flavia Guzzo

flavia.guzzo@univr.it

${ }^{\dagger}$ These authors have contributed equally to this work.

Specialty section:

This article was submitted to

Crop Science and Horticulture, a section of the journal

Frontiers in Plant Science

Received: 06 March 2017 Accepted: 26 May 2017

Published: 21 June 2017

Citation:

Negri S, Lovato A, Boscaini F, Salvetti E, Torriani S, Commisso M,

Danzi R, Ugliano M, Polverari A,

Tornielli GB and Guzzo F (2017) The

Induction of Noble Rot (Botrytis

cinerea) Infection during Postharvest

Withering Changes the Metabolome of Grapevine Berries Nitis vinifera L., CV. Garganega). Front. Plant Sci. 8:1002. doi: 10.3389/fpls.2017.01002

\section{The Induction of Noble Rot (Botrytis cinerea) Infection during Postharvest Withering Changes the Metabolome of Grapevine Berries (Vitis vinifera L., cv. Garganega)}

\author{
Stefano Negri ${ }^{1 \dagger}$, Arianna Lovato ${ }^{1 \dagger}$, Filippo Boscaini ${ }^{1}$, Elisa Salvetti ${ }^{1}$, Sandra Torriani ${ }^{1}$, \\ Mauro Commisso ${ }^{1}$, Roberta Danzi ${ }^{2}$, Maurizio Ugliano ${ }^{1}$, Annalisa Polverari ${ }^{1}$, \\ Giovanni B. Tornielli ${ }^{1 *}$ and Flavia Guzzo ${ }^{1 *}$
}

${ }^{1}$ Biotechnology Department, University of Verona, Verona, Italy, ${ }^{2}$ Unione Italiana Vini Soc. coop, Verona, Italy

The natural or induced development of noble rot caused by the fungus Botrytis cinerea during the late stages of grapevine (Vitis vinifera L.) berry ripening is used in some traditional viticulture areas to produce high-quality wines such as Sauternes and Tokaji. In this research, we wanted to verify if by changing the environmental conditions during post-harvest withering we could induce the noble rot development on harvested berries in order to positively change the wine produced from withered Garganega berries. Therefore, we exposed the berries to postharvest withering under normal or artificially humid conditions, the latter to induce noble rot. The presence of noble rot symptoms was associated with the development of $B$. cinerea in the berries maintained under humid conditions. The composition of infected and non-infected berries was investigated by untargeted metabolomics using liquid chromatography/mass spectrometry. We also explored the effects of the two withering methods on the abundance of volatile organic compounds in wine by yeast-inoculated micro-fermentation followed by targeted gas chromatography/mass spectrometry. These experiments revealed significant metabolic differences between berries withered under normal and humid conditions, indicating that noble rot affects berry metabolism and composition. As well as well-known botrytization markers, we detected two novel lipids that have not been observed before in berries infected with noble rot. Unraveling the specific metabolic profile of berries infected with noble rot may help to determine the compounds responsible for the organoleptic quality traits of botrytized Garganega wines.

Keywords: postharvest withering, Garganega grapes, noble rot induction, metabolomics, vOCs

\section{INTRODUCTION}

The necrotrophic ascomycete Botrytis cinerea has been described as a 'Jekyll and Hyde' fungus because it causes devastating gray mold disease in grapevine plants but is also responsible for noble rot in ripe and overripe berries, which allows the production of high-quality sweet wines such as Sauternes and Tokaji (Fournier et al., 2013). Gray mold caused by B. cinerea is one of the 
most severe grapevine diseases, reducing both the quality and quantity of berries. The resulting wines are poor because the infected berries have an unfavorable composition and the pathogen also produces toxic compounds that affect yeast and thus inhibit the fermentation process (Bocquet et al., 1995; Hong et al., 2011; Agudelo-Romero et al., 2015). However, the development of $B$. cinerea as noble rot (botrytization) is a favorable process lasting 10-20 days and is typical of particular wine productions. The berry is transformed by the penetration of fungi through stomata, wounds or microfissures on the fruit surface (the pourri plein stage), the permeabilization of the fruit skin encouraging water loss and sugar concentration, and finally enzymatic maceration (the pourri rôti stage) (RibéreauGayon et al., 2006). At the end of this process, further fungal development is arrested by the high sugar concentration and, if still on the plants, the botrytized berries can be harvested individually. Noble rot confers a berry composition which is distinct from that of berries with gray rot and uninfected berries and is potentially associated with desirable aroma characters of the resulting wine. In addition to directly producing potent odorants such as phenylacetaldehyde, lactones and vanillin (Lopez Pinar et al., 2016), noble rot infection can indeed stimulate production, in the berry, of cysteine and glutathione conjugates which can be transformed by the yeast into the powerful aroma compound 3-mercaptohexanol (Thibon et al., 2009, 2011). The developmental transition between gray rot and noble rot is influenced by environmental conditions and soil characteristics. Moist nights, foggy mornings and dry, sunny days promote the slow infection that results in noble rot, whereas strong rainfall and high humidity facilitate the more aggressive gray mold (Ribéreau-Gayon et al., 1980; Gubler et al., 2013).

Recioto di Soave is an Italian passito wine (i.e., a wine produced from dehydrated grapes) made from the white-skinned berries of the cultivar Garganega. Grape dehydration (known as withering) takes place after harvest in a dedicated room known as the fruttaio. Slow postharvest withering can favor noble rot development, induced by particular environmental conditions and/or artificial B. cinerea inoculation, thus allowing botrytization to be implemented in regions where natural noble rot is uncommon (Lorenzini et al., 2012; Tosi et al., 2013).

The metabolomic and transcriptomic changes that occur in black-skinned grape berries of various cultivars during traditional postharvest withering in fruttaio have recently been described (Zenoni et al., 2016). The behavior of withering berries is strongly cultivar dependent. The berries of slow-withering cultivars are more metabolically active during the process, showing both de novo synthesis of various metabolites (especially stilbenes) and a higher number of differentially expressed genes. Transcriptomics and metabolomics have also been used to investigate the response of white-skinned Sémillon berries during noble rot infections (Blanco-Ulate et al., 2015). The fruits of this cultivar respond to $B$. cinerea infection by upregulating genes involved in the response to pathogens and stress, fruit ripening, and hormone metabolism, and by accumulating certain secondary metabolites such as phenylpropanoids and terpenes.

Here we used untargeted metabolomics based on liquid chromatography/mass spectrometry (LC-MS) to characterize the metabolites of Garganega berries during postharvest withering in fruttaio, under standard conditions and with artificial humidification used to induce $B$. cinerea colonization. We also used targeted gas chromatography/mass spectrometry (GC-MS) to characterize the volatile organic compounds (VOCs) in wines produced from berries exposed to the two postharvest withering treatments.

\section{MATERIALS AND METHODS}

\section{Withering Methods and Berry Sampling}

Approximately $170 \mathrm{~kg}$ of Garganega berries was harvested at the commercial ripening stage (soluble solids content $=$ $18.5 \pm 0.25^{\circ}$ Brix) in Monteforte d'Alpone (Verona, Italy) at the beginning of October and transported to the Pasqua Vigneti e Cantine winery. The berries were placed in perforated plastic boxes (plateaux, $\sim 5 \mathrm{~kg}$ in each) in a ventilated withering facility under natural conditions $\left(17-20^{\circ} \mathrm{C}, 78-82 \%\right.$ relative humidity) and sampled (T0).

Brix degrees were measured weekly in three randomly selected replicates using a DBR35 digital refractometer (Giorgio Bormac, Carpi, Italy). Three boxes were also weighed weekly using a CH50K50 electronic balance (Kern, Balingen, Germany) in order to determine the weight loss of berry bunches during withering. After 29 days, when the weight loss was $\sim 30 \%$ of the initial weight, grape clusters were sampled (T1) and half of the plateaux were covered with plastic film at $15-17^{\circ} \mathrm{C}$. Water-filled trays were placed inside to increase the relative humidity $(88-94 \%)$ and encourage $B$. cinerea development. The remaining plateaux were left under normal withering conditions $\left(15-17^{\circ} \mathrm{C}, 68-75 \%\right.$ relative humidity). The two different environmental conditions were imposed for 32 days before the final samples were taken obtaining T2-n (normal withering, ventilated) and T2-i (induced noble rot) samples. The total duration of dehydration was 61 days. The relative humidity and temperature inside and outside the covering were monitored using Hobo Pro v2 sensors connected to data loggers (Onset Computer Corporation, Bourne, MA, USA).

Three independent pools of $3 \mathrm{~kg}$ of berries each were collected for each treatment and used in the following analysis. For T0, $\mathrm{T} 1$ and T2-n grape berries were randomly sampled whereas for T2-i berries were visually selected for noble rot symptoms. Each sample was used to determine the average berry weight, the number of $B$. cinerea colony forming units (CFUs) and for LCMS analysis. The remaining of T0, T2-n, and T2-i berry samples (about $2 \mathrm{Kg}$ for each biological replicates) were also pressed and the resulting musts were micro-fermented followed by GCMS analysis of the wine. A simplified experimental workflow is reported in Figure 1.

\section{Enumeration of $B$. cinerea CFUs}

We randomly selected $100 \mathrm{~g}$ of berries from each biological replicate and crushed them independently. The juices were serially diluted in 25\% Ringer's solution (Oxoid, Basingstoke, UK) and $100-\mu 1$ aliquots were spread on triplicate plates of Botrytis Selective Medium (Edwards and Seddon, 2001). The B. cinerea 


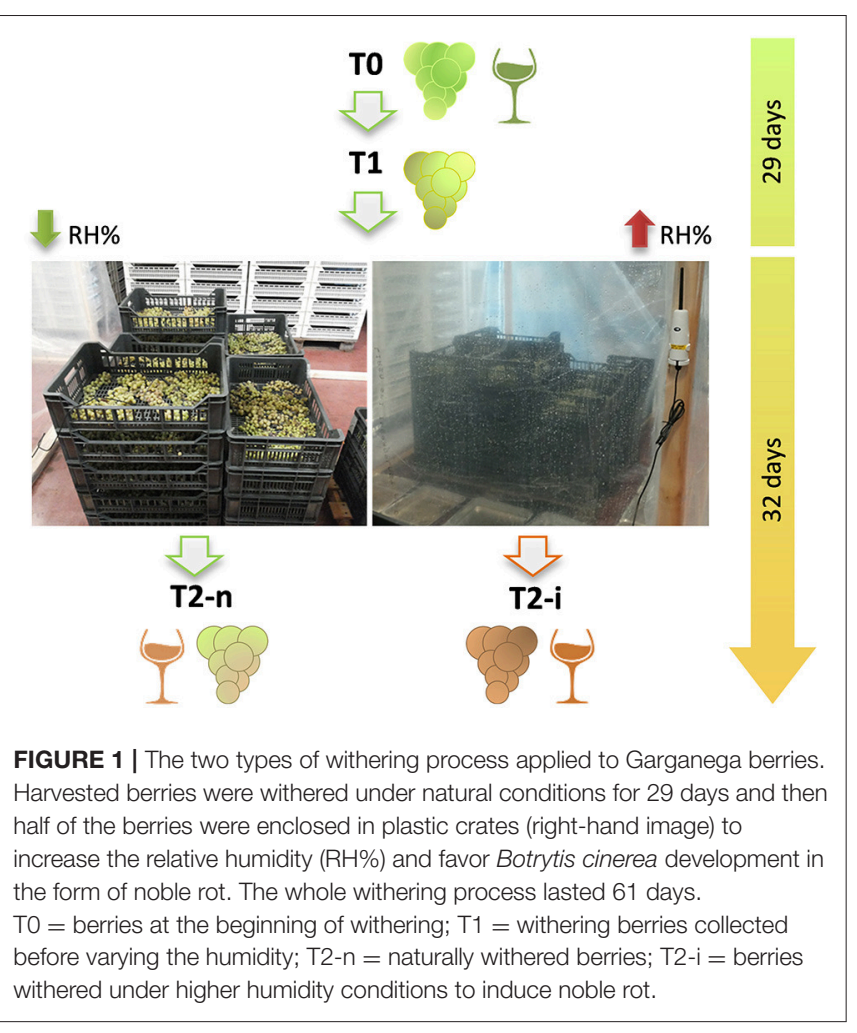

CFUs were counted on the plates after 5-7 days of incubation at $20^{\circ} \mathrm{C}$ in the dark.

\section{Extraction of Metabolites and LC-MS Analysis}

$250 \mathrm{~g}$ of frozen berries for each pool was ground in liquid nitrogen and $300 \mathrm{mg}$ of berry powder was extracted in three volumes of cold LC-MS-grade methanol. After mixing, the samples were sonicated for $15 \mathrm{~min}$ at $4^{\circ} \mathrm{C}$ and centrifuged (16 $000 \mathrm{rcf}, 10 \mathrm{~min}, 4^{\circ} \mathrm{C}$ ). The supernatants were diluted $1: 2$ in LC-MS-grade water, passed through $0.2 \mu \mathrm{m}$ Minisart RC4 filters (Sartorius-Stedim Biotech, Göttingen, Germany) and analyzed by reversed-phase high-performance liquid chromatography (RPHPLC) using a Gold 127 HPLC System (Beckman Coulter, Brea, CA, USA) equipped with a C18 guard column $(7.5 \times 2.1 \mathrm{~mm}$, $5 \mu \mathrm{m}$ particle size) and an Alltech (Nicholasville, KT, USA) RP C18 column $(150 \times 2.1 \mathrm{~mm}, 3 \mu \mathrm{m}$ particle size $)$. A gradient between solvent $\mathrm{A}$ ( $0.5 \%$ formic acid and $5 \%$ acetonitrile in water) and solvent B (100\% acetonitrile) was set as follows: $0-10 \% \mathrm{~B}$ in $2 \mathrm{~min}, 10-20 \% \mathrm{~B}$ in $10 \mathrm{~min}, 20-25 \% \mathrm{~B}$ in $2 \mathrm{~min}, 25-70 \% \mathrm{~B}$ in 7 $\mathrm{min}$, isocratic for $5 \mathrm{~min}, 70-90 \% \mathrm{~B}$ in $1 \mathrm{~min}$, isocratic for $14 \mathrm{~min}$, $90-0 \% \mathrm{~B}$ in $1 \mathrm{~min}$, and $20 \mathrm{~min}$ equilibration. For each sample, $20 \mu \mathrm{l}$ was injected at a flow rate of $0.2 \mathrm{ml} \mathrm{min}^{-1}$.

The HPLC instrument was coupled on-line to an Esquire 6000 ion trap mass spectrometer equipped with an electrospray ionization (ESI) source (Bruker Daltonik, Bremen, Germany). Mass spectra were recorded in alternating positive and negative ionization mode within the range $50-1,500 \mathrm{~m} / z$ with a target mass of $400 \mathrm{~m} / z$. Nitrogen was used as the nebulizing gas (50 psi, $\left.350^{\circ} \mathrm{C}\right)$ and drying gas $\left(10 \mathrm{~L} \mathrm{~min}^{-1}\right)$ and the vacuum pressure was $1.4 \times 10^{-8}$ bar. For fragmentation analysis, MS/MS and $\mathrm{MS}^{3}$ spectra were recorded in positive and negative ionization modes in the range $50-1,500 \mathrm{~m} / z$. Helium was used for collision induced dissociation (amplitude $=1 \mathrm{~V}$ ). MS data were collected using Esquire Control v5.2 software and processed using Esquire Data Analysis v3.2 software (both provided by Bruker Daltonik).

Metabolites were identified by comparing retention times, $\mathrm{m} / \mathrm{z}$ values and fragmentation patterns with those of commercial standards in our in-house library. When no authentic standard compounds were available, identification relied on the fragmentation patterns in online databases such as MassBank (www.massbank.jp) or reported in the literature. Neutral losses of 132, 146, and $162 \mathrm{Da}$ were considered diagnostic of the loss of pentose, deoxyhexose, and hexose sugars, respectively.

\section{Lipid Extraction and LC-MS Analysis}

Botrytis cinerea strain B05.10 (Amselem et al., 2011) was inoculated into flasks containing $125 \mathrm{ml}$ potato dextrose broth (Formedium, Hunstanton, UK) at a concentration of $7 \times 10^{6}$ conidia/ml, and incubated for 7 days at $22^{\circ} \mathrm{C}$, shaking at $120 \mathrm{rpm}$. $B$. cinerea mycelia were recovered by filtration and ground in liquid nitrogen. We resuspended $300 \mathrm{mg}$ of frozen berry powder or $B$. cinerea mycelia in $300 \mu \mathrm{l}$ LC-MS-grade water and then mixed the suspension with $3 \mathrm{ml}$ glacial chloroform/methanol $(2: 1)$. The samples were vortexed for $30 \mathrm{~s}$, stored on ice for $1 \mathrm{~h}$, sonicated for $15 \mathrm{~min}$ and centrifuged (25 min, 4,500 rcf, $\left.4^{\circ} \mathrm{C}\right)$. The chloroform phases $(\sim 2 \mathrm{ml})$ were recovered, placed in 2-ml plastic tubes and centrifuged again (10 min, 16,000 rcf, $4^{\circ} \mathrm{C}$ ). The supernatants were recovered, partially evaporated in a Heto Holton Maxi-Dry Plus Vacuum (Thermo Fisher Scientific, Waltham, MA, USA) and tubes containing the same extract were pooled. Finally, the solvent was completely evaporated, the residue was resuspended in three volumes $(\mathrm{w} / \mathrm{v})$ of LCMS-grade methanol and sonicated for $3 \mathrm{~min}$. One sample, arbitrary selected as Quality Control 1 (QC1), and a methanolic solution including $1 \mu \mathrm{g} / \mu \mathrm{l}$ palmitic acid as QC2 (Sigma-Aldrich, St Louis, MO, USA) were analyzed at the beginning and end of the experiment, respectively. Finally, the solutions were passed through a Minisart RC4 $0.2-\mu \mathrm{m}$ filter and $20 \mu \mathrm{l}$ was injected into the abovementioned LC-MS system. The solvents were $0.5 \%(\mathrm{v} / \mathrm{v})$ formic acid in LC-MS-grade water (A) and 100\% acetonitrile (B). A gradient was established from 50 to $100 \%$ B in $10 \mathrm{~min}$, followed by 65 min under isocratic conditions and then from 100 to $50 \%$ $\mathrm{B}$ in $1 \mathrm{~min}$. The column was finally equilibrated for $15 \mathrm{~min}$. MS analysis was carried out by equipping the mass spectrometer with an atmospheric pressure chemical ionization (APCI) source and using the same parameters described above for medium-polar metabolites.

\section{Micro-Vinification}

Fermentation trials were carried out using musts from T0, T2-n, and T2-i berry samples. The musts were separated from the pomace and mixed with $0.3 \mathrm{~g} / \mathrm{l}$ activating agent (Apapiù Mix, Tebaldi, Colognola ai Colli, Italy) and 15 $\mathrm{mg} / \mathrm{l}$ sodium metabisulfite (Sigma-Aldrich) before transferring $500 \mathrm{ml}$ of each must carefully into sterile bottles. After 
measuring the Brix degrees, the musts were inoculated with $10^{6} \mathrm{CFU} / \mathrm{ml}$ of the commercial wine strain Saccharomyces cerevisiae Mycoferm CRU 69, previously activated by following the manufacturer's instructions (Ever, Pramaggiore, Italy). Each fermentation experiment was performed in triplicate at a controlled temperature of $18^{\circ} \mathrm{C}$. We monitored the fermentation kinetics for 14 days by gravimetric analysis to determine the loss of weight due to the production of $\mathrm{CO}_{2}$.

\section{GC-MS Analysis of Micro-Vinificated Wines}

Volatiles were analyzed by gas chromatography-mass spectrometry (GC-MS) after solid-phase extraction (SPE). $\mathrm{SPE}$ was performed using $\mathrm{ENV}^{+}$cartridge $(1 \mathrm{~g}, 40-140 \mu \mathrm{m}$; Isolute, IST Ltd., Mid Glamorgan, UK) and an Aspec XL Sample Processor for SPE (Gilson Inc. Middleton, WI, USA). The cartridges were sequentially conditioned with methanol $(9.5 \mathrm{ml})$ and distilled water $(19 \mathrm{ml})$. A total of $38 \mathrm{ml}$ of wine sample diluted 1:2 with distilled water, and 1-heptanol added as internal standard $(500 \mu \mathrm{g} / \mathrm{l})$ was loaded onto the cartridge. The residue was washed with $19 \mathrm{ml}$ of distilled water. The free aroma compounds were eluted with $9 \mathrm{ml}$ of dichloromethane. The solution was dried with $\mathrm{Na}_{2} \mathrm{SO}_{4}$ and concentrated to $0.4 \mathrm{ml}$ by nitrogen flow stream. GC-MS analysis was performed with 6980N Network GC System coupled with a 5975 XL EI/CI MSD (Agilent Technologies, Santa Clara, CA, USA), equipped with DB-WAX Bonded PEG fused silica capillary column $(60 \mathrm{~m} \times 320 \mu \mathrm{m}$ i.d. $\times 0.25 \mu \mathrm{m}$ film thickness; Agilent Technologies). Instrumental conditions were: electron impact (EI) mode $70 \mathrm{eV}$; injector temperature $200^{\circ} \mathrm{C}$; He carrier flow $1.5 \mathrm{ml} / \mathrm{min}$; column temperature $50^{\circ} \mathrm{C}$ for $4 \mathrm{~min}$, rising to $240^{\circ} \mathrm{C}$ at $4^{\circ} \mathrm{C} / \mathrm{min}$, then $20 \mathrm{~min}$ at $240^{\circ} \mathrm{C}$; and injection volume $2.0 \mu \mathrm{L}$ in splitless mode. The analyses were performed in SCAN mode. NIST data bank and co-injection of pure reference standards were used to identify the compounds.

\section{Statistical Analysis of Samples}

Statistical significance between samples analyzed for percentage of weight loss, soluble solid content, average berry weight, $B$. cinerea enumeration and fermentation kinetics was evaluated by t-student. For LC-MS and GC-MS data, raw chromatograms were converted to netCDF files for peak alignment and area extraction using MZmine software (http://mzmine.sourceforge.net/) and multivariate statistical analysis was applied to the resulting dataset using SIMCA v.13.0 (UmetrixAB, Umeå, Sweden). Pareto scaling was applied to all analytical methods. Unsupervised principal component analysis (PCA) was used to identify the major clusters defined by the samples, and two supervised methods, namely partial least squares discriminant analysis (PLS-DA) and orthogonal projections to latent structures discriminant analysis (OPLSDA/O2PLS-DA), were used to compare classes in order to identify metabolites that characterize different withering stages. PLS-DA models were validated by a permutation test $(200$ permutations) and the corresponding OPLS-DA/O2PLS-DA models were cross-validated by analysis of variance (ANOVA) with a threshold of $p<0.01$.

\section{RESULTS}

\section{Artificial Humidification of Berries Increases the Prevalence of $B$. cinerea Colonization}

The berries maintained under standard conditions showed a linear weight loss during the first 40 days of withering followed by slower weight loss toward the end of the process, whereas the berries maintained under artificially high humidity lost weight more slowly ( $p<0.01$, Figure 2A). The Brix degrees increased during withering due to the loss of water and consequently the concentration of sugars, hence the profile was complementary to the loss of weight, increasing more slowly in the covered berries (Figure 2B).

At the end of the withering process, only the covered berries showed the typical symptoms of noble rot, with roughly $70 \%$ of the berries visually appearing as chocolate-brown colored, more shriveled and dehydrated compared to control berries (Figures 2C,D). These berries were visually selected for further analysis. The comparison between the average berry weight of visually selected T2-n and T2-i berries revealed that T2-i weight was slightly lower than the control berries $(p<0.05$, Figure 2E). This was also associated with a higher prevalence of $B$. cinerea colonization as determined by counting the number of CFUs on selective medium, confirming that the conditions used for induction supported $B$. cinerea growth in its latent form $(p<0.05$, Figure 2F). No gray mold symptoms were observed among the covered berries.

\section{Untargeted Metabolomics Reveals Fungal Metabolites, Plant Phytoalexin Accumulation, and Plant Metabolite Depletion}

Berries were sampled for metabolomic comparison at the beginning of the experiment (T0), 29 days later just before the two different withering conditions were applied (T1), and at the end of the experiment, separately for the berries exposed to conventional withering (T2-n) and the high humidity conditions (T2-i). Representative LC-MS chromatograms obtained in negative and positive ionization modes are shown in Figure 3. The blue zones highlight metabolites that increased in abundance during dehydration but were largely consumed in T2-i berries in comparison with T2-n berries. These metabolites included caffeoyl tartaric acid (caftaric acid), the amino acids leucine/isoleucine, phenylalanine, and tryptophan (together with its caffeic acid derivative), flavan-3-ols and flavonols. The red zones indicate metabolites that became more abundant or appeared de novo in the artificially humidified berries (T2-i) in comparison with $\mathrm{T} 2-\mathrm{n}$ berries. These included uridine 5'-diphospho-N-acetylglucosamine, a resveratrol tetramer and 13-keto-9Z,11E-octadecadienoic acid/13-oxo-9Z,11Eoctadecadienoic acid (13-KODE). The metabolites present in all samples are summarized in Table $\mathbf{1 .}$

The chromatograms were used to build two data matrices (negative and positive ionization mode, respectively). The negative ionization data matrix contained $257 \mathrm{~m} / \mathrm{z}$ features, 74 of 
A

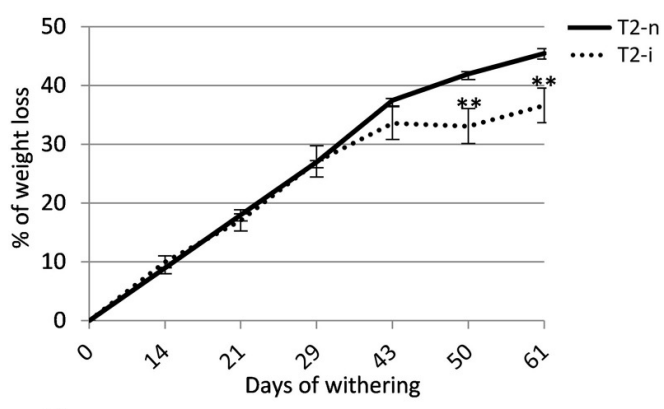

B

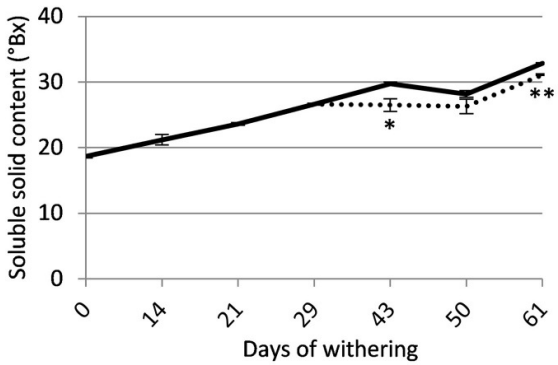

C

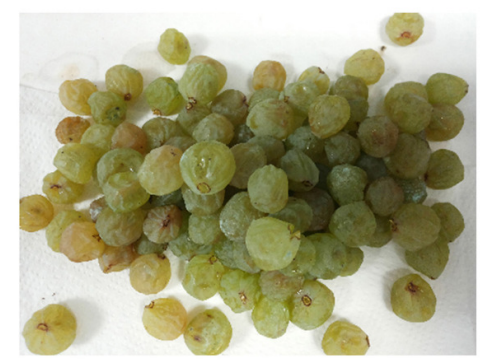

D

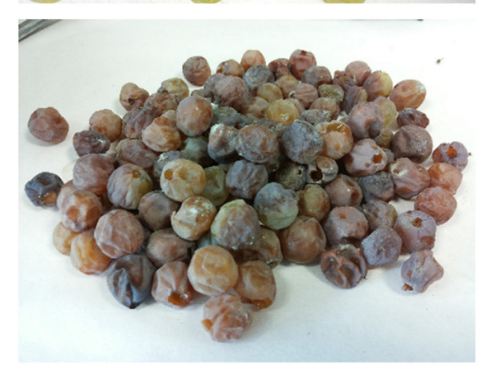

E

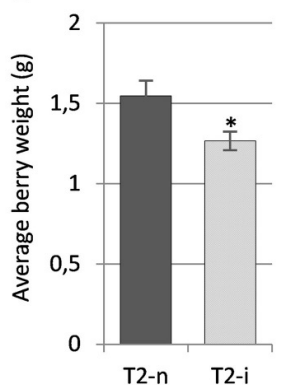

$\mathbf{F}$

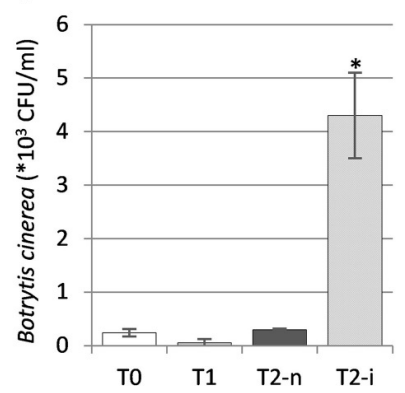

FIGURE 2 | Garganega berry characteristics. (A) Percentage weight loss and (B) soluble solids $\left({ }^{\circ} \mathrm{Bx}\right)$ during normal withering $(\mathrm{T} 2-\mathrm{n})$ and induced noble rot (T2-i). (C) Appearance of sampled berries withered under natural conditions or (D) under higher humidity to induce noble rot. (E) Average berry weight in the T2-n and T2-i samples at the end of the withering process. (F) Enumeration of Botrytis cinerea colony forming units (CFUs) in samples T0, T1, T2-n, and T2-i. Vertical bars represented standard deviations (SD) of means $(n=3)$. Asterisks refer to $t$-student $p$-values obtained from T2-n and T2-i comparison $\left({ }^{*} p<0.05,{ }^{* *} p<0.01\right)$. which were tentatively identified. They included 52 metabolites plus adducts and fragments (Supplementary File 1, Datasheet 1). The positive ionization data matrix contained $356 \mathrm{~m} / z$ features, 33 of which were identified, corresponding to 22 different metabolites (Supplementary File 1, Datasheet 2).

The two data matrices were explored by multivariate analysis (O2PLS-DA). The results obtained for the negative ionization data matrix are shown in Supplementary Figures 1A,B. The O2PLS-DA loading plot was expressed as a pq(corr) value, representing the correlation between the $p$ part of the model (the class of samples) and the q part of the model (the metabolites). The spatial closeness among the metabolites (black triangles) and the samples (blue squares) reflects their relationships, revealing the concentration effect from $\mathrm{T} 0$ to $\mathrm{T} 1$ and $\mathrm{T} 2$, but also specific effects in the botrytized samples that differ substantially from the non-botrytized controls (Supplementary Figure 1B). To overcome concentration effects and focus on concentrationindependent effects, the data matrices were normalized for weight loss, and the signal intensities were expressed relative to the weight at the beginning of the experiment (Supplementary File 1, Datasheets 3, 4). The results following data normalization are shown for the negative data matrix (Figures $4 \mathbf{A}, \mathbf{B}$ ). The enrichment of metabolites previously observed in the T2-n samples is now effectively shared among the T0, T1, and T2$\mathrm{n}$ samples (light blue circle) confirming these metabolites are characteristic of the entire traditional withering process. Despite this normalization effect, a few metabolites were typical of the T0 and T2-n control samples (green and orange circles, respectively). Interestingly, the botrytized berries (brown circles) were strongly characterized by a group of specific metabolites which correlate negatively with the traditional withering process. The metabolites are listed in Supplementary File 2, Datasheet 1.

OPLS-DA analysis was also applied to both the negative and positive ionization mode data matrices for the normal withering (T2-n) and botrytized (T2-i) berry samples (Figures 4C,D) better highlighting differences between T2-n and T2-i. The set of strongly characteristic metabolites identified by this analysis (Supplementary File 2, Datasheet 2) reflects the depletion and de novo production of metabolites in T2-i as already highlighted in Figure 3 and reported in Table 1 (e.g., resveratrol derivatives, 13-KODE and uridine $5^{\prime}$-diphospho- $\mathrm{N}$-acetylglucosamine).

The depleted compounds represent diverse metabolite classes (e.g., sugars, amino acids, flavonoids and some stilbenes). Other metabolites accumulate rapidly in the botrytized berries, including pantothenic acid, some stilbenes (dimers, trimers and tetramers but not the monomers), glucose-6-phosphate, uridine $5^{\prime}$-diphospho-N-acetylglucosamine, a lipid putatively annotated as $13-\mathrm{KODE}$, and other unidentified metabolites. The presence of the $\mathrm{N}$-acetylglucosamine donor uridine $5^{\prime}$-diphospho- $\mathrm{N}$ acetylglucosamine indicates an active fungal metabolism because this sugar is a precursor of the chitin found in the fungal cell wall. Some of the molecules accumulating in botrytized berries were also clearly detectable in the chromatograms as major signals (Figure 3), including one resveratrol tetramer (Figure 3) and two metabolites with retention times of 30 and 32 min respectively (not shown). These two metabolites showed similar behavior, suggesting similar chemical properties. Both showed the chloride and formic adducts as main signals in negative ionization mode, 


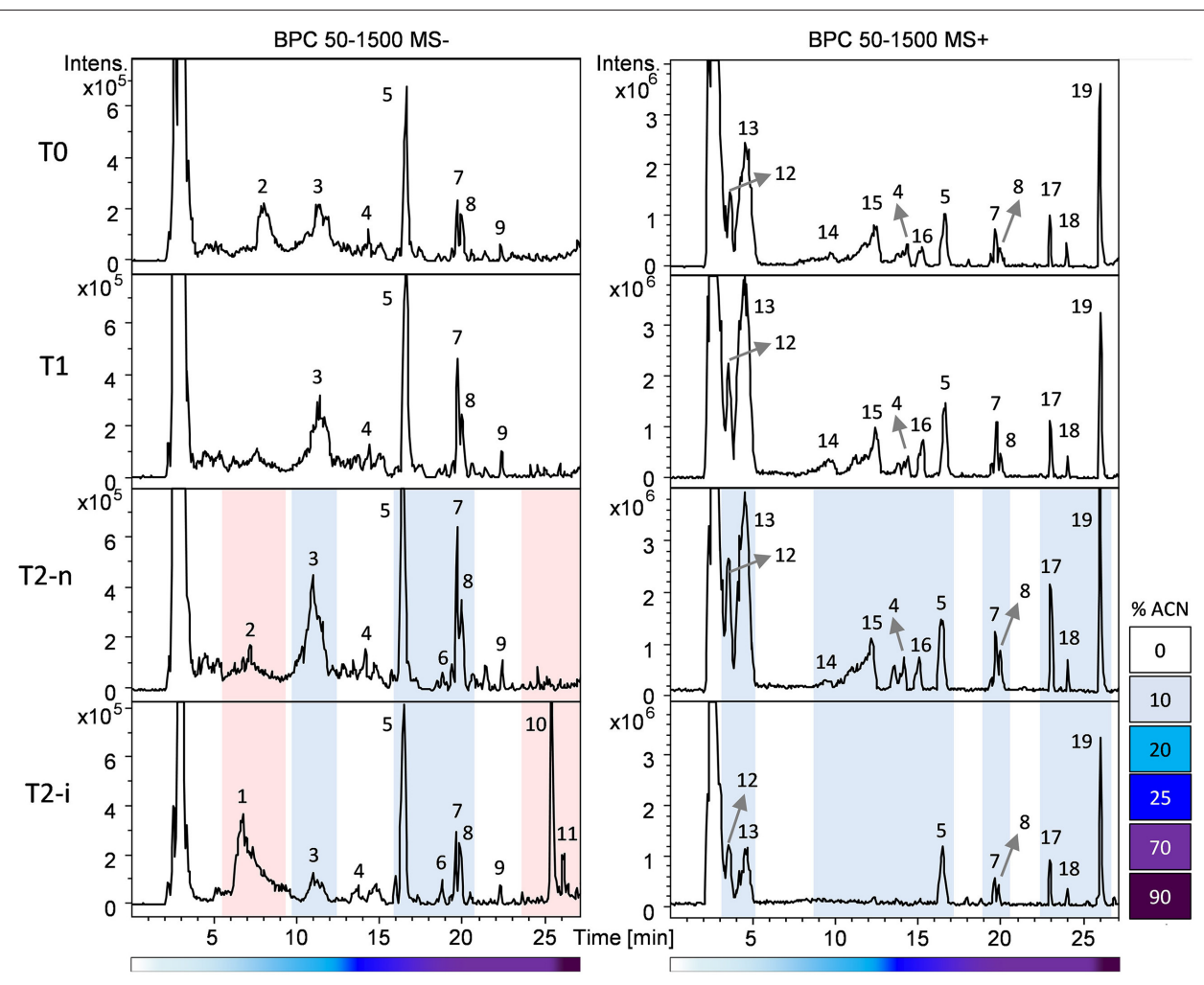

FIGURE 3 | Base peak chromatograms (BPC) recorded in negative (left) and positive (right) ionization mode based on the RP-HPLC-ESI-MS analysis of T0, T1, T2-n, and T2-i berry samples. Peak numbers refer to the metabolites listed in Table 1. Chromatogram areas are highlighted to show the reduction (in blue) or the accumulation (in red) of metabolites in T2-i grapes relative to naturally withered grapes (TO, T1, T2-n). The lower bars refer to the percentage of acetonitrile (ACN) in the mobile phase.

and the molecular ions in positive ionization mode, and both generated fragments at $m / z 355,337$, and 206. Although we were unable to identify these molecules, the higher retention time suggested they are lipids. We therefore re-extracted the methanol extracts with chloroform, and the resulting lipid fractions were analyzed by LC-APCI-MS using a method optimized for lipid analysis. The lipid profiles of T2-n and botrytized (T2-i) samples are shown in Supplementary Figure 2. This analysis confirmed the lipid-like nature of the two unidentified T2-i metabolites (highlighted in the figure) and showed that the general lipid profile is otherwise similar between the T2-n and T2-i samples. The same peaks could not be detected using the same LC-APCIMS approach following the extraction of lipids from $B$. cinerea strain B05.10 grown in vitro, indicating that the two unidentified lipids might not be typical constituents of the fungus (data not shown). On the other side, we cannot exclude that the wild type $B$. cinerea strains developed in this experiments have different composition than the used reference B05.10 strain (Amselem et al., 2011).

\section{The Wines Produced by Botrytized and Conventionally Withered Berries Show Different Aromatic Profiles}

Micro-scale vinification was performed on fresh berries (T0), withered botrytized berries (T2-i) and conventionally withered berries (T2-n). The ${ }^{\circ}$ Brix values of the musts from the T0, $\mathrm{T} 2-\mathrm{i}$ and $\mathrm{T} 2-\mathrm{n}$ berries were $18.67 \pm 0.25,31.13 \pm 0.11$, and $32.87 \pm 0.06$, respectively. The must fermentation rate (calculated as grams of $\mathrm{CO}_{2} / 100 \mathrm{ml}$ of must) was generally higher in the musts from withered grapes (Figure 5A). Moreover, musts from the conventionally withered grapes showed a more vigorous fermentation compared to the musts from botrytized berries $(p<0.05)$.

The aromatic profile of the three wines was analyzed by GC-MS 14 days after the beginning of the vinification. The O2PLS-DA model of the entire GC-MS data matrix revealed that the three wines showed distinct aromatic compositions (Figures 5B,C). The compounds characterizing the three wines are listed in Table 2, and their pq(corr) values are shown in Supplementary File 3. Wines from T2-n grapes were mainly characterized by fruity aromas, whereas botrytized wines were characterized by spicy aromas (Figure 5C).

When the two withering processes were compared, wine from the naturally withered berries was strongly characterized by the presence of ethyl-4-hydroxy butanoate, as well as benzyl alcohol, eugenol, guaiacol, homovanillic alcohol, homovanillic acid, trans-3-hexenol, $\beta$-damascenone, and methyl vanillate. In contrast, the botrytized wine was strongly characterized by the presence of $\mathrm{N}$-(3-methylbutyl)acetamide, as well as sherry lactone 1, benzaldehyde, 1-octen-3-ol, trans-8-dihydroxylinalool, 
TABLE 1 | Main metabolites detected by RP-HPLC-ESI-MS analysis in negative $\left([\mathrm{M}-\mathrm{H}]^{-}\right)$and positive $\left([\mathrm{M}+\mathrm{H}]^{+}\right)$ionization modes.

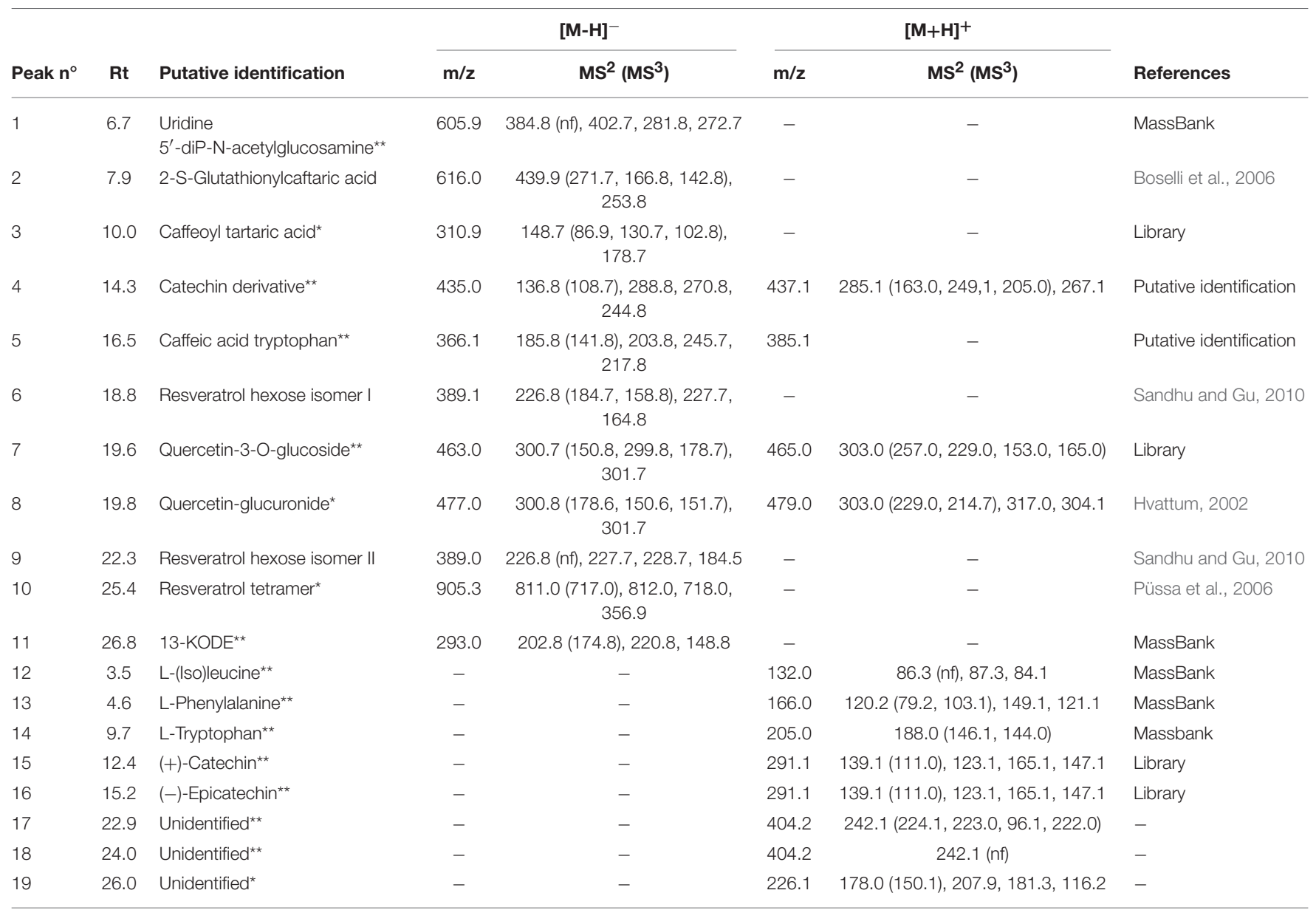

Peak numbers refer to the chromatogram profiles in Figure 3. Rt, retention time (min); Nf, not fragmented. Asterisks refer to those metabolites which average peak areas was significantly different between T2-n and T2-i as assessed by $t$-student test.

${ }^{*} p<0.05,{ }^{* *} p<0.01$.

ethyl vanillate, ethyl isoamyl succinate, diethyl succinate, p-cresol, ho-diendiol, 4-terpineol, $\gamma$-nonalactone, and ethyl phenylacetate (Figure 5D and Supplementary File 3). When considering only the more abundant VOCs (more than $500 \mathrm{ppb}$ ) showing at least a two-fold difference in abundance between the two samples, the wine produced from naturally-withered berries was characterized by isovalerianic acid, isoamylacetate, decanoic acid, and homovanillic acid, whereas the botrytized wine was characterized by $\mathrm{N}$-(3-methylbutyl)-acetamide, sherry lactones 1 and 2, benzaldehyde and 4-terpineol.

\section{DISCUSSION}

\section{The Natural Development of Noble Rot Can Be Strongly Induced in Garganega Berries Undergoing Postharvest Dehydration}

The postharvest induction of noble rot could be used for the production of botrytized wines in regions with climates unsuitable for natural botrytization and also in those with suitable climates, to overcome the unpredictability of natural botrytization. However, a controlled widespread noble rot development on dehydrating grapes is not easy to achieve because the natural or forced ventilation of cases to accelerate dehydration makes the berries less susceptible to infection with B. cinerea (Barbanti et al., 2008). Fedrizzi et al. (2011) investigated natural botrytization in Corvina berries during withering, but in this case it was necessary to discard rotten berries developing gray mold and to manually separate the botrytized and non-botrytized fruit. Lorenzini et al. (2012) showed that noble rot can be induced under postharvest laboratory conditions by inoculating Garganega and Corvina berries with the fungus. The ability to achieve widespread noble rot development during natural withering has been reported anecdotally (Ferrarini et al., 2009; Vannini and Chilosi, 2013).

Here we demonstrated the ability to induce noble rot development in Garganega berries without the concomitant development of gray mold by implementing a special management strategy during postharvest withering, comprising an initial period of normal withering to allow partial berry dehydration (which prevents the development of gray mold by ensuring the adequate concentration of sugars) followed by a 

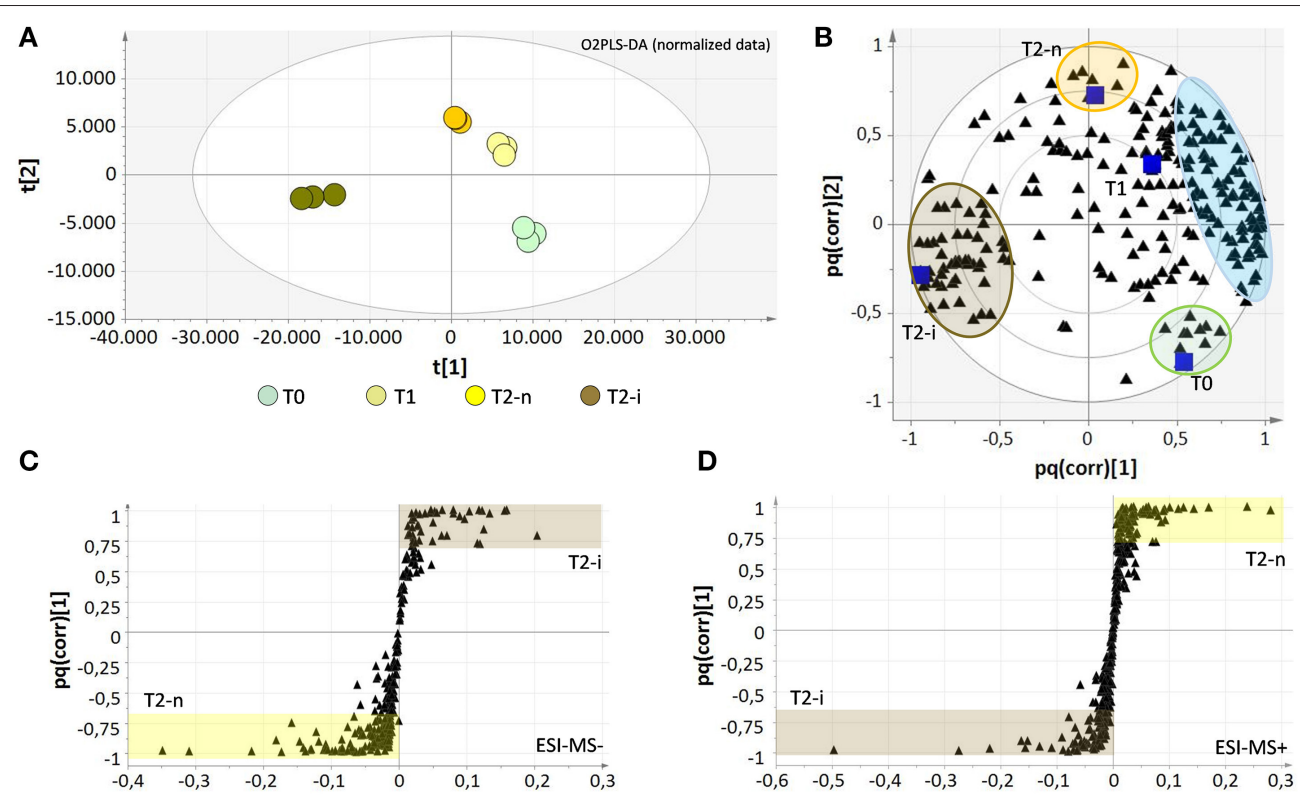

FIGURE 4 | Score plots (A) and correlation loading plots (B) for the O2PLS-DA model of the negative data matrices after data normalization for sample weight loss. The metabolites which strongly characterize each sample are highlighted with colored circles and are listed in Supplementary File 2 (Datasheet 1). The light blue circle comprises all metabolites that characterize the natural withering process and negatively correlate with berries infected with noble rot (T2-i). Correlation loading plots for the OPLS-DA models of negative (C) and positive (D) data matrices show the distribution of metabolites between T2-n and T2-i berries. All metabolites with pq(corr) values $>0.7$ or $<-0.7$ are considered highly characteristic of T2-n (highlighted in yellow) or T2-i (highlighted in brown) berries and are listed in the Supplementary File 2 (Datasheet 2).

period of increased humidity achieved by covering the berries in the presence of water-filled trays. This simple procedure increased the humidity without affecting the temperature, slightly reduced the rate of berry dehydration, and encouraged the development of $B$. cinerea infection without the need for artificial inoculation because the fungus is commonly present in the vineyard and in cellars as an environmental contaminant. Noble rot induction was confirmed by berry characteristics and the enumeration of $B$. cinerea CFUs in selective medium. We characterized the changes in the metabolite profile of grapes and wines attributable to the proliferation of the fungus. However, the possibility that the modified air humidity could be the cause of part of the differences between T2-n and T2-i cannot be completely ruled out.

\section{B. cinerea Growth and Plant Defence Can Be Monitored by Untargeted Metabolomics}

Untargeted metabolomics based on LC-MS revealed metabolites associated with the infection of berries by $B$. cinerea. Some of these metabolites were biochemical markers of the fungus, including structural components and products of fungal metabolism, while others were derived from the berries and represent the onset of plant defense mechanisms during withering.

The LC-MS data matrix revealed many imprints of fungal metabolism, including the presence of the $\mathrm{N}$-acetylglucosamine donor uridine $5^{\prime}$-diphospho- $\mathrm{N}$-acetylglucosamine, which is utilized by fungi including $B$. cinerea as a substrate for the enzyme chitin synthase (Causier et al., 1994). The declining levels of many grape metabolites in botrytized fruits suggests they were degraded by fungal metabolism, including sucrose, hydroxycinnamic acids (coutaric, caftaric, and fertaric acids), amino acids, lignans, and many flavonoids (including flavan-3ols and flavonols). The loss of polyphenols has been reported in other white-berry cultivars infected with noble rot, including Chenin Blanc (Carbajal-Ida et al., 2016) and Chardonnay (Hong et al., 2012) although there was a specific increase in the abundance of flavan-3-ols in Chenin Blanc, in contrast to other polyphenols (Carbajal-Ida et al., 2016). However, cultivars such as Sémillon accumulated high levels of phenylpropanoids following the onset of noble rot (Blanco-Ulate et al., 2015). Therefore, the impact of $B$. cinerea on phenylpropanoid metabolism appears to be cultivar dependent.

The stilbenes are phytoalexins that are known to accumulate during botrytization (Landrault et al., 2002; Blanco-Ulate et al., 2015). The observed decline in the abundance of stilbene monomers (resveratrol and resveratrol glucoside) could reflect the consumption of these metabolites by the fungus, but the concomitant increase in the levels of stilbene dimers, trimers and tetramers suggests that botrytization causes the aggregation of stilbene monomers into oligomers. The accumulation of the oxylipin 13-KODE could also represent a plant defense response because this metabolite is induced as a defense molecule in soybean (Glycine max) in response to fungi such as Aspergillus niger, A. oryzae, Rhizopus oligosporus, and A. niger wry (Feng et al., 2007). To the best of our knowledge, this is the first 

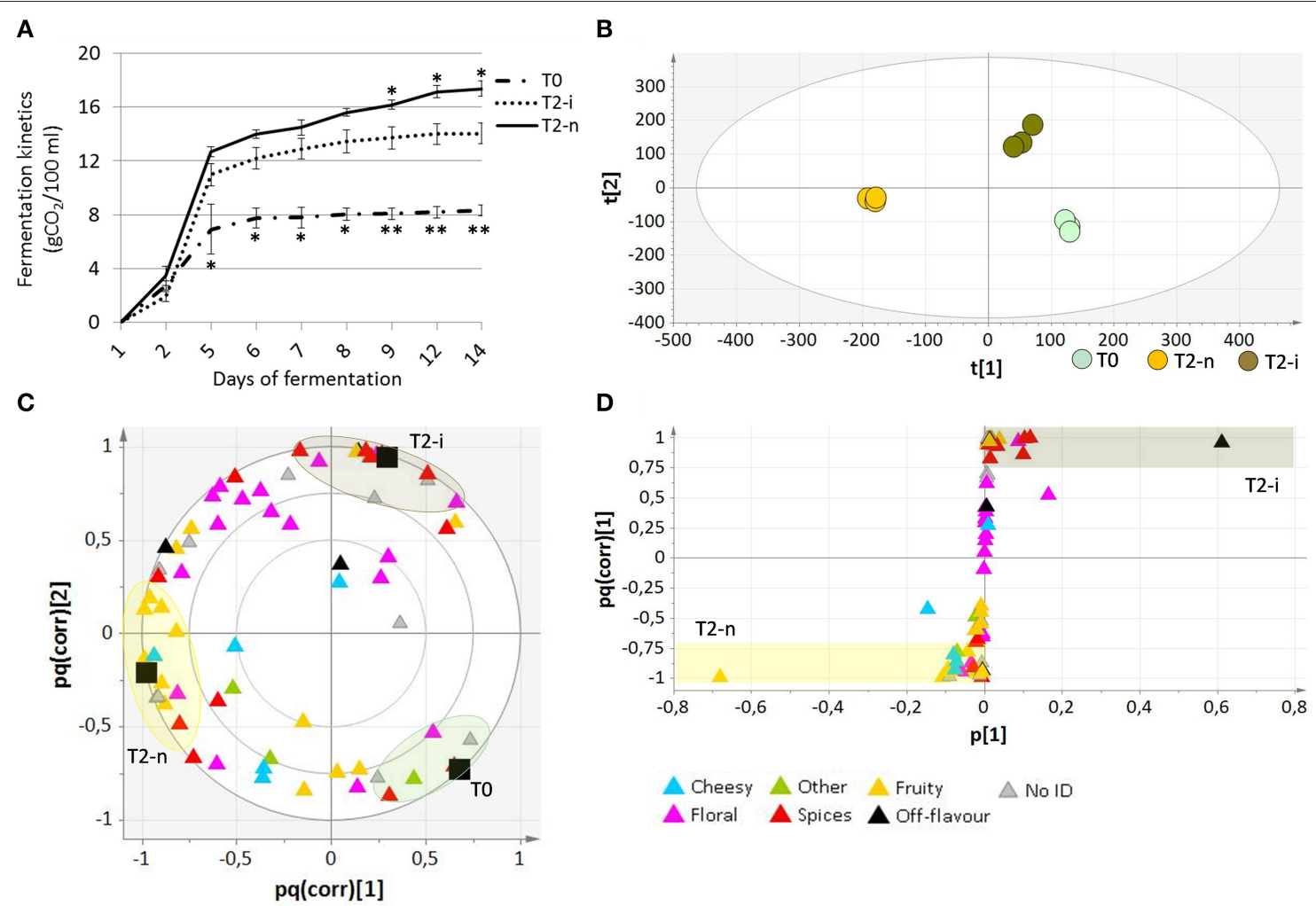

FIGURE 5 | Analysis of wines produced from Garganega berries. (A) Fermentation kinetics of the musts obtained from T0, T2-n, and T2-i berries during the 14 days of micro-vinification. Vertical bars represented standard deviations (SD) of means $(n=3)$. Asterisks refer to $t$-student $p$-values obtained from comparison of T2-i with T-n and T0 samples ( ${ }^{\star} p<0.05,{ }^{\star *} p<0.01$ ) (B) O2PLS-DA score plot and (C) loading plot of T0, T2-n, and T2-i wines. In (C) correlation loading of the aroma differentiation characterizing each sample is represented by a color code, with VOCs grouped according to their aromatic class. (D) OPLS-DA loading correlation plot of withered control (T2-n) and botrytized (T2-i) wines.

report describing the induction of 13-KODE in grapevine berries in response to noble rot. However, octadecadienoic acids, the precursors of KODE oxylipins, have been proposed as potential positive metabolic markers of gray mold (Agudelo-Romero et al., 2015). The botrytized fruits also accumulated large amounts of pantothenic acid, D-glucose-6-phosphate and two unannotated lipids.

\section{The Postharvest Induction of Noble Rot Influenced the Accumulation of Wine Aroma Compounds}

The proliferation of $B$. cinerea induced remarkable changes in the accumulation of VOCs, affecting several aroma compounds that may contribute to the sensory characters of white wines. From a quantitative perspective, $\mathrm{N}$-(3-methylbutyl)acetamide was the strongest marker of botrytized wine in agreement with previous studies of botrytization in Recioto di Soave (Azzolini et al., 2013; Tosi et al., 2013), Amarone (Fedrizzi et al., 2011), and Fiano (Genovese et al., 2007) wines. From a qualitative perspective, several VOCs detected at lower concentrations but with a potentially higher impact on aroma (Francis and Newton, 2005) were also influenced by noble rot. The sherry lactone isomers and $\gamma$-nonalactone were detected at higher concentrations in botrytized wines, in agreement with previous reports (Genovese et al., 2007; Sarrazin et al., 2007; Azzolini et al., 2013; Tosi et al., 2013). Although lactones do not contribute directly to the aroma of botrytized wines, they are involved in perceptive interaction phenomena resulting in an enhanced sensory contribution, e.g., synergy between $\gamma$-nonalactone and eugenol can enhance the overripe orange aroma notes typical of noble rot wines (Stamatopoulos et al., 2014). Eugenol is mostly derived from contact between the wine and oak wood, so the storage in oak barrels of Garganega wines from berries infected with noble rot could enhance these overripe orange aromas. Terpenes such as citronellol, ho-diendiol, hydroxylated linalool derivatives and 4-terpineol became more abundant during dehydration in the berries with noble rot, especially in the case of 4-terpineol. Likewise, the norisoprenoid 3-oxo- $\alpha$-ionol (which gives rise to the tobacco aroma compound megastigmatrienone) accumulated to higher levels during withering. Terpenes and norisoprenoids are two important groups of aroma compounds that contribute the floral, fruity and tobacco-like attributes of wines. They accumulate in the berries as free molecules and as glycosylated precursors, which can be revealed by the action of yeast during fermentation or by acid hydrolysis during wine aging (Ugliano 
TABLE 2 | Aroma compounds highlighted in Figure 5C characterizing T0, T2-n, and T2-i musts.

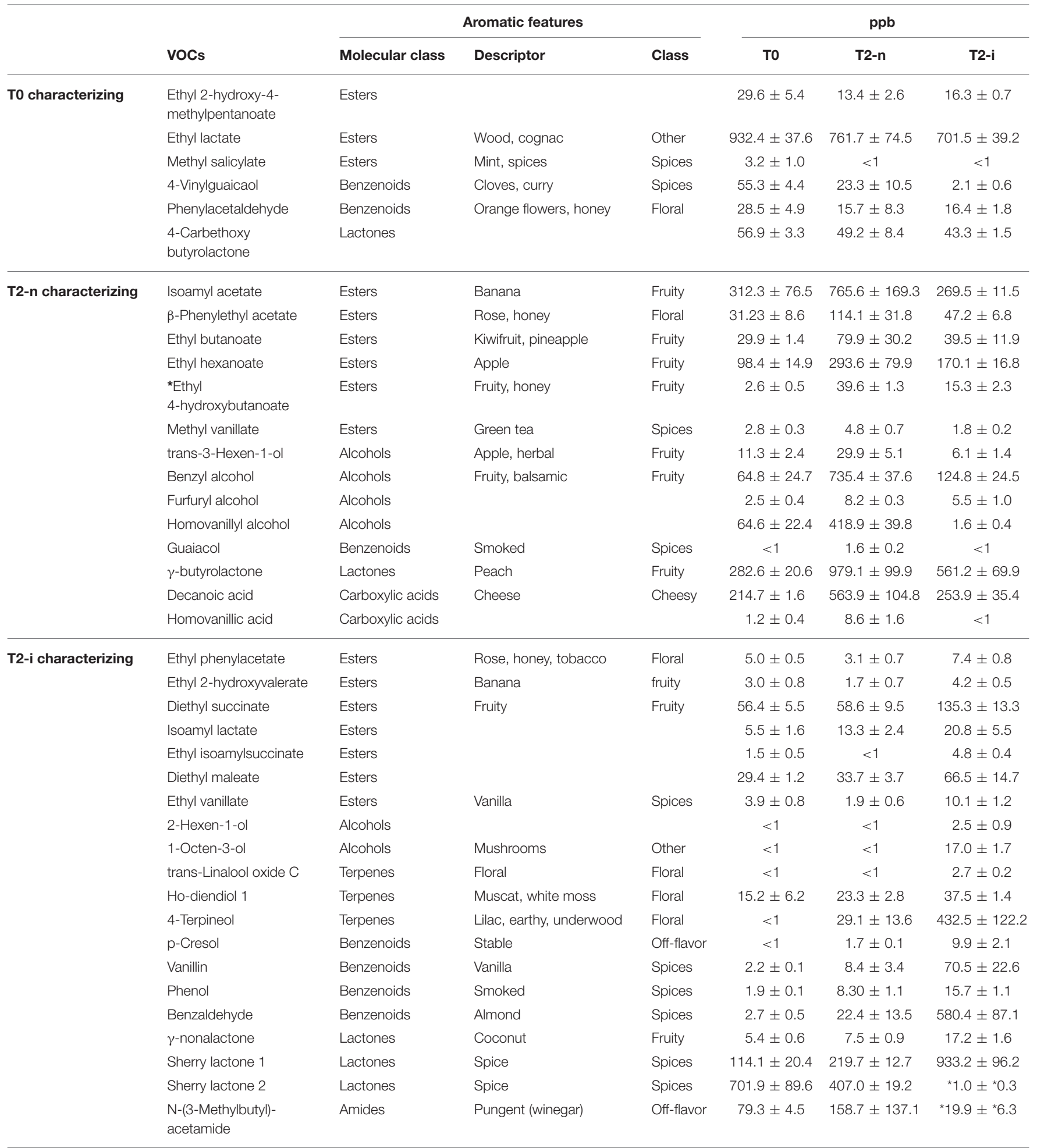

The corresponding pq(corr) values are reported in Supplementary File 3. Items marked with an asterisk have to be multiplied by $10^{3}$.

et al., 2006). Dehydration can favor their accumulation, and the presence of $B$. cinerea can facilitate their release from precursors by means of the pool of enzymes released into the must (Donèche, 1993).
Several volatile benzenoids, generally characterized by sweet/spicy aroma notes, were shown to increase in response to noble rot and/or simple dehydration, including benzaldehyde, vanillin, cresols, guaiacols and eugenol. Although benzaldehyde 
is often associated with the development of B. cinerea (Genovese et al., 2007; Fedrizzi et al., 2011), the behavior of volatile benzenoids has not been investigated in detail. However, Genovese et al. (2007) also observed the accumulation of eugenol and vinyl guaiacol in white wines prepared from berries infected with noble rot. The mushroom-like aroma compound 1-octen-3-ol is found at significantly higher concentrations in wines produced from botrytized berries. B. cinerea and other pathogens such as Uncinula necator (powdery mildew) produce 1-octen-3-ol, which at high concentrations can reduce the quality of berries and introduce mushroom off-odors in the finished wine (Darriet et al., 2002).

Interestingly, several aroma compounds arising from yeast metabolism, in particular the powerful fruit-smelling esters isoamyl acetate, ethyl butanoate, ethyl hexanoate and ethyl octanoate, were present at lower concentrations in the botrytized wines compared to wines from either the fresh or dehydrated berries. When comparing fresh and dehydrated berries, it was clear that dehydration without noble rot infection favored the accumulation of these metabolites during fermentation, probably due to the higher concentrations of nitrogen available to the yeast (Ugliano et al., 2006; Vilanova et al., 2007). The lower concentrations of esters in the botrytized wines could therefore reflect the depletion of nitrogen or the release of esterases by B. cinerea.

In conclusion, the analysis of the volatile fraction of wines and evaluation of the potential odor contribution of different volatiles indicated that wines from dehydrated berries were generally characterized by higher content of fresh fruit-smelling compounds (esters), whereas noble rot induced the accumulation of several spicy aroma compounds such as lactones, combined with compounds with floral attributes such as 4-terpineol and the mushroom smelling compound 1-octen-3-ol.

\section{REFERENCES}

Agudelo-Romero, P., Erban, A., Rego, C., Carbonell-Bejerano, P., Nascimento, T., Sousa, L., et al. (2015). Transcriptome and metabolome reprogramming in Vitis vinifera cv. Trincadeira berries upon infection with Botrytis cinerea. J. Exp. Bot. 66, 1769-1785. doi: 10.1093/jxb/eru517

Amselem, J., Cuomo, C. A., Van Kan, J. A., Viaud, M., Benito, E. P., Couloux, A., et al. (2011). Genomic analysis of the necrotrophic fungal pathogens Sclerotinia sclerotiorum and Botrytis cinerea. PLoS Genet. 7:e1002230. doi: 10.1371/journal.pgen.1002230

Azzolini, M., Tosi, E., Faccio, S., Lorenzini, M., Torriani, S., and Zapparoli, G. (2013). Selection of Botrytis cinerea and Saccharomyces cerevisiae strains for the improvement and valorization of Italian passito style wines. FEMS Yeast Res. 13, 540-552. doi: 10.1111/1567-1364.12054

Barbanti, D., Mora, B., Ferrarini, R., Tornielli, G. B., and Cipriani, M. (2008). Effect of various thermo-hygrometric conditions on the withering kinetics of grapes used for the production of "Amarone" and "Recioto" wines. J. Food Eng. 85, 350-358. doi: 10.1016/j.jfoodeng.2007.07.003

Blanco-Ulate, B., Amrine, K. C., Collins, T. S., Rivero, R. M., Vicente, A. R., Morales-Cruz, A., et al. (2015). Developmental and metabolic plasticity of white-skinned grape berries in response to Botrytis cinerea during noble rot. Plant Physiol. 169, 2422-2443. doi: 10.1104/pp.15.00852

Bocquet, F., Moncomble, D., and Valade, M. (1995). Etat sanitaire de la vendange et qualité des vins. Le Vigneron Champenois 7, 15-23.

\section{AUTHOR CONTRIBUTIONS}

GT: designed the experiments; SN and FB: performed the LCMS-based untargeted metabolomics; $\mathrm{SN}$ and RD: performed the GC-MS based metabolomics; MC: performed the lipidomic experiment; ST and ES: did the bacterial counts and analyses; $\mathrm{AP}$ and AL: projected and executed the sampling and the agronomical analyses; SN and FG: analyzed the metabolomics data; FG: wrote the manuscript; SN, AL, MU, and GT: contributed to the draft writing; MU, AP, and ST: critically revised the manuscript; all authors read and approved the final manuscript.

\section{FUNDING}

This work was supported by Regione Veneto - POR - Fondo Sociale Europeo 2007-2013 - Ob. Competitività Regionale e Occupazione - Reg. 1081/2006. Asse IV “Capitale Umano." This work benefited from the networking activities coordinated within the EU-funded COST ACTION FA1106 "An integrated systems approach to determine the developmental mechanisms controlling fleshy fruit quality in tomato and grapevine."

\section{ACKNOWLEDGMENTS}

We are grateful to the winery "Pasqua Vigneti e Cantine SpA" for providing Garganega samples and for allowing us to set up the experiment in their withering facility.

\section{SUPPLEMENTARY MATERIAL}

The Supplementary Material for this article can be found online at: http://journal.frontiersin.org/article/10.3389/fpls.2017. 01002/full\#supplementary-material

Boselli, E., Minardi, M., Giomo, A., and Frega, N. G. (2006). Phenolic composition and quality of white doc wines from Marche (Italy). Anal. Chim. Acta 563, 93-100. doi: 10.1016/j.aca.2005.10.024

Carbajal-Ida, D., Maury, C., Salas, E., Siret, R., and Mehinagic, E. (2016). Physico-chemical properties of botrytised Chenin blanc grapes to assess the extent of noble rot. Eur. Food Res. Technol. 242, 117-126. doi: 10.1007/s00217-015-2523-x

Causier, B. E., Milling, R. J., Foster, S. G., and Adams, D. J. (1994). Characterization of chitin synthase from Botrytis cinerea. Microbiology 140, 2199-2205. doi: 10.1099/13500872-140-9-2199

Darriet, P., Pons, M., Henry, R., Dumont, O., Findeling, V., Cartolaro, P., et al. (2002). Impact odorants contributing to the fungus type aroma from grape berries contaminated by powdery mildew (Uncinula necator); incidence of enzymatic activities of the yeast Saccharomyces cerevisiae. J. Agric. Food Chem. 50, 3277-3282. doi: 10.1021/jf011527d

Donèche, B. J. (1993). "Botrytized wines," in Wine Microbiology and Biotechnology, ed G. H. Fleet (Chur; New York, NY: Harwood Academic Publishers), 327-353.

Edwards, S. G., and Seddon, B. (2001). Selective media for the specific isolation and enumeration of Botrytis cinerea conidia. Lett. Appl. Microbiol. 32, 63-66.

Fedrizzi, B., Tosi, E., Simonato, B., Finato, F., Cipriani, M., Caramia, G., et al. (2011). Changes in wine aroma composition according to botrytized berry percentage: a preliminary study on Amarone wine. Food Technol. Biotechnol. $49,529-535$. 
Feng, S., Saw, C. L., Lee, Y. K., and Huang, D. (2007). Fungal-stressed germination of black soybeans leads to generation of oxooctadecadienoic acids in addition to glyceollins. J. Agric. Food Chem. 55, 8589-8595. doi: 10.1021/jf0716735

Ferrarini, R., Casarotti, E. M., Zanella, G., and Nicolis, E. (2009). Botrytis cinerea noble form induction on grapes during withering. Am. J. Enol. Vitic. 60:399A.

Fournier, E., Gladieux, P., and Giraud, T. (2013). The "Dr Jekyll and Mr Hyde fungus": noble rot versus gray mold symptoms of Botrytis cinerea on grapes. Evol. Appl. 6, 960-969. doi: 10.1111/eva.12079

Francis, I. L., and Newton, J. L. (2005). Determining wine aroma from compositional data. Aust. J. Grape Wine Res. 11, 114-126. doi: 10.1111/j.1755-0238.2005.tb00283.x

Genovese, A., Gambuti, A., Piombino, P., and Moio, L. (2007). Sensory properties and aroma compounds of sweet Fiano wine. Food Chem. 103, 1228-1236. doi: 10.1016/j.foodchem.2006.10.027

Gubler, W. D., Hashim, J. M., Smilanick, J. L., and Leavitt, G. M. (2013). “Gray mold (Botrytis cinerea)," in Grape Pest Management, Vol. 3343, ed L. J. Bettiga (UCANR Publications), 133-136.

Hong, Y. S., Cilindre, C., Liger-Belair, G., Jeandet, P., Hertkorn, N., and Schmitt-Kopplin, P. (2011). Metabolic influence of Botrytis cinerea infection in champagne base wine. J. Agric. Food Chem. 59, 7237-7245. doi: $10.1021 /$ jf $200664 t$

Hong, Y. S., Martinez, A., Liger-Belair, G., Jeandet, P., Nuzillard, J. M., and Cilindre, C. (2012). Metabolomics reveals simultaneous influences of plant defence system and fungal growth in Botrytis cinerea-infected Vitis vinifera cv. Chardonnay berries. J. Exp. Bot. 63, 5773-5785. doi: 10.1093/jxb/ers228

Hvattum, E. (2002). Determination of phenolic compounds in rose hip (Rosa canina) using liquid chromatography coupled to electrospray ionisation tandem mass spectrometry and diode-array detection. Rapid Commun. Mass Spectrom. 16, 655-662. doi: 10.1002/rcm.622

Landrault, N., Larronde, F., Delaunay, J. C., Castagnino, C., Vercauteren, J., Merillon, J. M., et al. (2002). Levels of stilbene oligomers and astilbin in French varietal wines and in grapes during noble rot development. J. Agric. Food Chem. 50, 2046-2052. doi: 10.1021/jf010794g

Lopez Pinar, A., Rauhut, D., Ruehl, E., and Buettner, A. (2016). Effects of Botrytis cinerea and Erysiphe necator fungi on the aroma character of grape must: a comparative approach. Food Chem. 207, 251-260. doi: 10.1016/j.foodchem.2016.03.110

Lorenzini, M., Azzolini, M., Tosi, E., and Zapparoli, G. (2012). Postharvest grape infection of Botrytis cinerea and its interactions with other moulds under withering conditions to produce noble-rotten grapes. J. Appl. Microbiol. 114, 762-770. doi: 10.1111/jam.12075

Püssa, T., Floren, J., Kuldkepp, P., and Raal, A. (2006). Survey of grapevine Vitis vinifera stem polyphenols by liquid chromatography- diode array detection-tandem mass spectrometry. J. Agric. Food Chem. 54, 7488-7494. doi: $10.1111 /$ jam. 12075

Ribéreau-Gayon, J., Ribérau-Gayon, P., and Seguin, G. (1980). “Botrytis cinerea in enology," in The Biology of Botrytis, eds J. R. Coley-Smith, K. Verhoeff, and W. R. Jarvis (London: Academic Press), 251-274.

Ribéreau-Gayon, P., Dubourdieu, D., Donèche, B., and Lonvaud-Funel, A. (2006). Handbook of Enology: The Microbiology of Wine and Vinifications, 2nd Edn. Chichester: Wiley and Sons.
Sandhu, A. K., and Gu, L. (2010). Antioxidant capacity, phenolic content, and profiling of phenolic compounds in the seeds, skin, and pulp of Vitis rotundifolia (muscadine grapes) as determined by HPLC-DAD-ESI-MS $\mathrm{n}$. J. Agric. Food Chem. 58, 4681-4692. doi: 10.1021/jf904211q

Sarrazin, E., Dubourdieu, D., and Darriet, P. (2007). Characterization of key-aroma compounds of botrytized wines, influence of grape botrytization. Food Chem. 103, 536-545. doi: 10.1016/j.foodchem.2006.08.026

Stamatopoulos, P., Frérot, E., Tempère, S., Pons, A., and Darriet, P. (2014). Identification of a new lactone contributing to overripe orange aroma in Bordeaux dessert wines via perceptual interaction phenomena. J. Agric. Food Chem. 62, 2469-2478. doi: 10.1021/jf405397c

Thibon, C., Cluzet, S., Mérillon, J. M., Darriet, P., and Dubourdieu, D. (2011). 3-Sulfanylhexanol precursor biogenesis in grapevine cells: the stimulating effect of Botrytis cinerea. J. Agric. Food Chem. 59, 1344-1351. doi: 10.1021/ jf103915y

Thibon, C., Dubourdieu, D., Darriet, P., and Tominaga, T. (2009). Impact of noble rot on the aroma precursor of 3-sulfanylhexanol content in Vitis vinifera $\mathrm{L}$. cv. Sauvignon blanc and Semillon grape juice. Food Chem. 114, 1359-1364. doi: 10.1016/j.foodchem.2008.11.016

Tosi, E., Azzolini, M., Lorenzini, M., Torriani, S., Fedrizzi, B., Finato, F., et al. (2013). Induction of grape botrytization during withering affects volatile composition of Recioto di Soave, a "passito"-style wine. Euro. Food Res. Technol. 236, 853-862. doi: 10.1007/s00217-013-1943-8

Ugliano, M., Bartowsky, E. J., McCarthy, J., Moio, L., and Henschke, P. A. (2006). Hydrolysis and transformation of grape glycosidically bound volatile compounds during fermentation with three Saccharomyces yeast strains. J. Agric. Food Chem. 54, 6322-6331. doi: 10.1021/jf0607718

Vannini, A., and Chilosi, G. (2013). "Botrytis infection: grey mould and noble rot," in Sweet, Reinforced and Fortified Wines: Grape Biochemistry, Technology and Vinification, eds F. Mencarelli and P. Tonutti (Hoboken, NJ: Wiley-Blackwell), 159-169.

Vilanova, M., Ugliano, M., Varela, C., Siebert, T., Pretorius, I. S., and Henschke, P. A. (2007). Assimilable nitrogen utilisation and production of volatile and non-volatile compounds in chemically defined medium by Saccharomyces cerevisiae wine yeasts. Appl. Microbiol. Biotechnol. 77, 145-157. doi: 10.1007/s00253-007-1145-z

Zenoni, S., Fasoli, M., Guzzo, F., Dal Santo, S., Amato, A., Anesi, A., et al. (2016). Disclosing the molecular basis of the postharvest life of berry in different grapevine genotypes. Plant Physiol. 172, 1821-1843. doi: 10.1104/pp.16.00865

Conflict of Interest Statement: The authors declare that the research was conducted in the absence of any commercial or financial relationships that could be construed as a potential conflict of interest.

Copyright (c) 2017 Negri, Lovato, Boscaini, Salvetti, Torriani, Commisso, Danzi, Ugliano, Polverari, Tornielli and Guzzo. This is an open-access article distributed under the terms of the Creative Commons Attribution License (CC BY). The use, distribution or reproduction in other forums is permitted, provided the original author(s) or licensor are credited and that the original publication in this journal is cited, in accordance with accepted academic practice. No use, distribution or reproduction is permitted which does not comply with these terms. 